\title{
Esports Agents with a Theory of Mind: Towards Better Engagement, Education, and Engineering
}

\author{
Murtuza N. Shergadwala \\ mshergad@ucsc.edu \\ University of California, Santa Cruz \\ Santa Cruz, California, USA
}

\author{
Magy Seif El-Nasr \\ mseifeln@ucsc.edu \\ University of California, Santa Cruz \\ Santa Cruz, California, USA
}

\begin{abstract}
The role of $\mathrm{AI}$ in esports is shifting from leveraging games as a testbed for improving AI algorithms to addressing the needs of the esports players such as enhancing their gaming experience, esports skills, and providing coaching. For AI to be able to effectively address such needs in esports, AI agents require a theory of mind, that is, the ability to infer players' tactics and intents. To that end, in this position paper, we argue for human-in-the-loop approaches for the discovery and computational embedding of the theory of mind within behavioral models of esports players. We discuss that such approaches can be enabled by player-centric investigations on situated cognition that will expand our understanding of the cognitive and other unobservable factors that influence esports players' behaviors. We conclude by discussing the implications of such a research direction in esports as well as broader implications in engineering design and design education.
\end{abstract}

\section{CCS CONCEPTS}

- Human-centered computing $\rightarrow$ User models; HCI theory, concepts and models.

\section{KEYWORDS}

theory of mind, esports, player modeling, player cognition, humanin-the-loop

\section{ACM Reference Format:}

Murtuza N. Shergadwala and Magy Seif El-Nasr. 2021. Esports Agents with a Theory of Mind: Towards Better Engagement, Education, and Engineering. In CHI 2021: ACM CHI Conference on Human Factors in Computing Systems, May 08-13, 2021, Yokohama, Japan. ACM, New York, NY, USA, 4 pages. https://doi.org/10.1145/nnnnnnn.nnnnnnn

\section{AI IN ESPORTS}

The use of AI technologies to enhance gaming experience of esports players is booming [33]. Such technologies span player-centric use cases such as enabling players to automatically import other expert player's team compositions[34], providing them their game play statistics, and strategy suggestions to maximize their learning potential [29]. AI agents are also being used as decision-support

Permission to make digital or hard copies of all or part of this work for personal or classroom use is granted without fee provided that copies are not made or distributed for profit or commercial advantage and that copies bear this notice and the full citation on the first page. Copyrights for components of this work owned by others than ACM must be honored. Abstracting with credit is permitted. To copy otherwise, or republish, to post on servers or to redistribute to lists, requires prior specific permission and/or a fee. Request permissions from permissions@acm.org.

CHI 2021, May 08-13, 2021, Yokohama, Japan

(C) 2021 Association for Computing Machinery.

ACM ISBN 978-x-xxxx-xxxx-x/YY/MM...\$15.00

https://doi.org/10.1145/nnnnnnn.nnnnnnn tools to generate team compositions to enable players to practice with different teams as well as predict success metrics and win probabilities [19, 20].

Although current AI technologies have advanced to provide game analytics [13] to esports players, the player-centric needs in esports go beyond suggesting heuristics and enabling team compositions [43]. Such needs include gaining skills such as understanding players' gameplay strategies, feeling a degree of sharedness in strategy with other team members, being able to infer opposing team's strategic plans, and to identify and act on opportunities within diverse situational contexts. Currently, players hope to satisfy such needs by gaining expertise through practice with human competitors not AI agents [15]. Freeman and Wohn [15] discuss that the desire to play with human players by esports players stems from the technical concern of AI agents lacking versatility. Such versatility includes the humanistic ability to interpret others' intentions, beliefs, knowledge, and mental states from observable data which is referred to as the theory of mind [32].

In the context of esports environments, typically characterized as multi-agent environments, the theory of mind is routinely leveraged by human players to reason about other players who may be teammates or competitors. Understanding others' intents and plan can be crucial to both cooperate and compete at the same time. In order for AI agents to facilitate such understanding, they too would require the theory of mind. Thus, developing computational esports agents with a theory of mind has not only the potential to alleviate the current concerns by esports players about AI agents [15] but also has several benefits such as improving player engagement, performance, and collaboration skills [8]. Moreover, esports agents with a theory of mind act as testbeds for broader impact in intelligent tutoring for education [26], discovery of human expertise in collaboration [31] which in turn would influence collaboration in engineering design teams [23], and development of theories for human-AI collaboration [41].

Endowing esports agents with a theory of mind is challenging due to several reasons. First, it is challenging to make inferences on unobservable factors such as intents and beliefs of others [5]. Due to the inherent unobservability of such aspects, there is a lack of quantitative data as well as approaches to generate such data about player intent, beliefs, and desires. Second, computational models of the theory of mind $[3,46]$ are in their infancy and cannot be directly applied in the context of esports due to the sheer complexity of esports environments. This includes players making inferences about multiple unobservable aspects such as individual-level beliefs and intents, team-level beliefs and intents, as well as situational inferences for opportunistic planning. Third, there is a lack of esports 
player-centric investigations that can shed light on the mental processes of players that implicitly leverage such unobservable factors to influence players' gameplay actions [30]. Thus, there is a need to investigate cognitive and situational factors that influence esports players' gameplay behaviors towards understanding their theory of mind.

In this position paper, we argue for human-in-the-loop (HITL) approaches for player-centric investigations towards developing computational models of esports agents with a theory of mind. We expand on this position statement such that we discuss (1) the current approaches for developing computational models of esports player behaviors and the research gaps towards understanding esports players' situated cognition, (2) the need for HITL approaches that integrate qualitative and quantitative inputs into the computational process of modeling intent, strategies, and tactics as a graphical probabilistic model, and (3) the implications of such approaches on esports and other broader implications.

\section{CURRENT APPROACHES TO MODELING ESPORTS PLAYER BEHAVIOR}

Player modeling, also termed as opponent modeling in some contexts [40], refers to the abstraction of a player's state, characteristics, and behaviors in the game [24, 40]. There also exists a taxonomy for the characteristics of the player that are modeled which includes a player's knowledge, position, strategy, and satisfaction about the game [24, 25]. The emphasis of modeling such player characteristics using AI agents has primarily been on generating believable behaviors [1] towards enabling human-AI interaction, improving player engagement, and customizing a game to address player needs [7, 16, 33]. However, there is a lack of focus on player models that abstract player cognition and mental processes.

Approaches to player modeling, broadly fall under model-based, model-free, and hybrid approaches [44]. Model-based approaches leverage theory mostly pertaining to human emotions for generating believable behaviors [45]. Model-free approaches assume some unknown functional mapping and leverage machine learning approaches such as Reinforcement Learning (RL) and Neural Networks to mimic player behaviors using data. Hybrid approaches leverage a combination of theory and data to model player behaviors [24]. Recently, researchers extended RL models to represent dynamic decision-making processes within complex stochastic environments [22]. Further, OpenAI made great strides in beating DotA2 human players using RL [9]. Such AI agents have been built using hybrid approaches and have been able to win games against human players. However, esports players' needs go beyond just winning the game to needs such as wanting to improve their gameplay skills [15].

Improvement in esports skills requires coaching in the form of detection and explanation of strategies from observation of players' gameplay. While current advancements in player modeling can lead to complex and robust agents, they are limited in how they can be used to detect and explain tactics and intent from observed real-world human behaviors [27]. This is because such models lack representation of unobservable factors that influence player behavior such as their cognition and prior knowledge. Model-free approaches for player behavior do account for unobservable factors through latent parameter modeling [42], such parameters are typically arbitrary and lack physical significance to the actual gameplay to derive meaningful explanations of player intent or strategies. Such abilities are the core competencies of the theory of mind, which is crucial for esports players to collaborate with team members as well as develop counter strategies to defeat opposing teams. Moreover, such detection and explanation of strategies is essential for esports coaching.

Existing literature in computational cognitive psychology has demonstrated the benefits of computationally embedding the theory of mind to address topics such as plan recognition [6], belief modeling [4], and intent recognition [39]. Such works demonstrate the ability to perform mental simulations of others' situation-dependent activities. While such works have made progress towards enabling a computational approach to the theory of mind, its application within esports is limited.

Computationally embedding the theory of mind in esports agent is still challenging due to several research gaps. First, there is a lack of player-centric investigations on the unobservable factors that players leverage while making decisions in esports gameplay. Such investigation will need to adopt HITL approaches to probe the mental processes of esports players. Second, in the context of esports, understanding intent and strategies is further complicated as players replan, behavior correct, and adopt opportunistic behaviors. Third, situational variations in esports environments are plenty and there is a lack of framework that enables capturing such variations in esports. To address such gaps, in the following section we argue for HITL approaches and elaborate how it would enable embedding theory of mind in esports agents.

\section{TOWARDS A THEORY OF MIND IN ESPORTS: THE CASE FOR HUMAN-IN-THE-LOOP APPROACHES}

HITL approaches imply leveraging cognitive power of humans to improve computational models [28]. Such approaches are crucial for complex situations where human expertise is required to make inferences [21]. HITL approaches enable development of socially intelligent agents, that is, agents with theory of mind capabilities [11]. HITL is also foundational in areas such as Interactive Machine Learning [14] to improve algorithmic predictive accuracy. Another area of relevance is open-learner models [12] where learners are the HITL who interact with computational models towards improving their understanding, involvement, and interaction with the learning environment. We assert that such HITL approaches can also be leveraged within esports to develop AI agents with a theory of mind that is capable of inferring intents and strategies of players and enabling esports coaching.

In the context of esports, we envision players would integrate qualitative and quantitative inputs into the computational models of esports players as a graphical probabilistic model. We argue that HITL approaches will enable players to interactively correct the computational model's probabilities as well as nodes that make up the graphical model. Questions remain on how HITL approaches could manifest in esports context such as, do we need to interrupt players or would players provide feedback in retrospection. We believe that context specific case studies would provide a better 
understanding of how to leverage HITL approaches. For example, in a tutorial mode, a player could be interrupted to receive as well as provide feedback to an "AI coach". Whereas, in a live game, interruption may be infeasible and possibly detrimental to a player's experience. Currently, however, there is lack of frameworks, methods, and infrastructure to enable players-in-the-loop to encapsulate their thoughts computationally.

In order to facilitate HITL approaches in esports, we require player-centric investigations on cognitive factors and mechanisms that influence players' gameplay. Cognitive factors here refer to the unobservable mental processes of players that influence their gameplay. Player-centric investigation refer to uncovering such cognitive factors. Existing literature in esports recognizes the need for such investigations as well $[17,30]$. Player-centric cognitive investigations would enable the development of a cognitive taxonomy of esports players. Such a taxonomy would provide different classes of cognitive variables and situational contexts that can be leveraged as blueprints to create a computational infrastructure for efficiently enabling HITL approaches to infer and embed cognitive and unobservable factors. For example, let's suppose that a player-centric investigation reveals that a player's perception about an opponent's aggressive or defensive behavior influences their gameplay strategy. In such an example, a cognitive variable would imply computationally representing such player perception within a class of similar perceptions of the player about their opponent. In other words, player-centric investigations of cognitive mechanisms of their gameplay would provide a structure for HITL approaches to efficiently embed player's theory of mind in AI agents.

For cognitive investigations in esports, we argue for a situated cognition perspective. Situated cognition posits that knowing is inseparable from doing [10] such that knowledge resides within the situations and activities bound to social, physical and cultural contexts. In the context of esports, such a view is relevant because leveraging the players to make inferences about mental states would deepen our understanding of how such players infer intent and tactics as well as improve their gameplay skills by encountering variations in gameplay situation.

Our HITL approach for cognitive investigations of esports players is to leverage players to label mental states of observed behaviors using Stratmapper [2]- our visualization system designed to visualize user actions, such as moving the mouse on an interface or map. Further, StratMapper allows us to filter events and use freeform text to label behaviors indicating the time and spatial contexts. Using the generated labels, we will apply a qualitative content analysis method to categorize various cognitive and situational factors such as player attention and intent. Moreover, expert players will be interviewed about their labeling choices to further understand the cognitive mechanisms. We note that we do not prescribe our approach for investigating cognitive mechanisms of player behaviors. Rather we emphasize the need for understanding cognitive mechanisms in esports by developing HITL approaches in esports.

We emphasize that player-centric investigations of cognitive mechanisms acts as a stepping stone towards enabling HITL approaches for embedding theory of mind in computational agents. Further challenges remain such as developing the computational infrastructure to efficiently capture the intricacies of situational contexts and behavioral variations in esports, addressing scalability to enable a player community to contribute to the computational models, and developing validation approaches to evaluate the impact of such models on esports player outcomes.

\section{IMPLICATIONS OF EMBEDDING THEORY OF MIND IN ESPORTS}

In this position paper, we highlight the need to embed theory of mind capabilities of players within AI models to enable reasoning about players or other AI agents in the game environment. We argue for HITL approaches that combine computation and cognition such that the computational representation of mental states is reflective of player cognition and not an arbitrary representation of latent variables. We discuss the implications of such research both within the context of esports and the broader implications in serious games for education and engineering design in the context of human-AI collaboration.

\subsection{Within Esports}

Endowing esports agents with a theory of mind will result in a paradigm-shift in the way esports player interact with AI agents which could manifest in novel innovative gameplay experiences. Beyond player-agent interaction, AI agents would act as intelligent tutoring systems for esports that would improve player expertise and skills in a personalized manner beyond simple heuristics and other player specific strategies. Such AI agents would improve believability of the gameplay experience, thereby, impacting game narratives and creative story telling as well.

\subsection{Broader Implications: Engineering Design and Design Education}

While the implications of AI agents with a theory of mind on humancomputer interaction are plenty, we focus on the impact of esports research on engineering design and design education. We highlight the similarity of spatio-temporal interaction in esports with engineering applications such as Computer Aided Design (CAD) as well as the similarity of esports player behaviors to engineering designers.

In esports, players collaborate in teams, make sequential and dynamic decisions, interact with their spatial environment, and their strategies evolve temporally as the gameplay proceeds. Similarly, engineering design teams collaboratively make domain-specific decisions [18] that involve interacting with a design environment and their strategies evolve as they move from conceptual design stage to the later stages of an engineering design process [38]. We note that domain knowledge and problem-specific aspects of the engineering design process influence design outcomes [35, 37]. Such aspects are difficult to teach as a part of the engineering education curricula [36]. The understanding of the design of AI agents with a theory of mind that would assist esports players in discovering strategies could also be leveraged by engineering design teams. Such teams would collaborate better by understanding individual team member's approach towards problem solving facilitated by the AI agent. Moreover, such agents could act as intelligent tutors for engineering design students to enable them explicitly understand the impact of their procedural decision making and problem solving strategies on design outcomes. 


\section{ACKNOWLEDGEMENT}

The authors gratefully acknowledge research support by a Seed Fund Award (2020-0139) from CITRIS and the Banatao Institute at the University of California.

\section{REFERENCES}

[1] Nuno Afonso and Rui Prada. 2008. Agents that relate: Improving the social believability of non-player characters in role-playing games. In International Conference on Entertainment Computing. Springer, Berlin, Heidelberg, 34-45.

[2] Sabbir Ahmad, Andy Bryant, Erica Kleinman, Zhaoqing Teng, Truong-Huy D Nguyen, and Magy Seif El-Nasr. 2019. Modeling Individual and Team Behavior through Spatio-Temporal Analysis. In Proceedings of the Annual Symposium on Computer-Human Interaction in Play (Barcelona, Spain) (CHI PLAY '19). Association for Computing Machinery, New York, NY, USA, 601-612. https://doi.org/10.1145/3311350.3347188

[3] Chris Baker, Rebecca Saxe, and Joshua Tenenbaum. 2011. Bayesian theory of mind: Modeling joint belief-desire attribution. In Proceedings of the annual meeting of the cognitive science society, Vol. 33 . Retrieved from https://escholarship.org/uc/item/5rk7z59q

[4] Chris Lawrence Baker. 2012. Bayesian theory of mind: Modeling human reasoning about beliefs, desires, goals, and social relations. Ph.D. Dissertation. Massachusetts Institute of Technology.

[5] Chris L Baker, Rebecca Saxe, and Joshua B Tenenbaum. 2009. Action understanding as inverse planning. Cognition 113, 3 (2009), 329-349.

[6] Chris L Baker and Joshua B Tenenbaum. 2014. Modeling human plan recognition using Bayesian theory of mind. Plan, activity, and intent recognition: Theory and practice 7 (2014), 177-204.

[7] Sander CJ Bakkes, Pieter HM Spronck, and Giel van Lankveld. 2012. Player behavioural modelling for video games. Entertainment Computing 3, 3 (2012), 71-79.

[8] Nolan Bard, Jakob N Foerster, Sarath Chandar, Neil Burch, Marc Lanctot, H Francis Song, Emilio Parisotto, Vincent Dumoulin, Subhodeep Moitra, Edward Hughes, et al. 2020. The hanabi challenge: A new frontier for ai research. Artificial Intelligence 280 (2020), 103216.

[9] Christopher Berner, Greg Brockman, Brooke Chan, Vicki Cheung, Przemysław Dębiak, Christy Dennison, David Farhi, Quirin Fischer, Shariq Hashme, Chris Hesse, et al. 2019. Dota 2 with large scale deep reinforcement learning. arXiv preprint arXiv:1912.06680 (2019).

[10] John Seely Brown, Allan Collins, and Paul Duguid. 1989. Situated cognition and the culture of learning. Educational researcher 18, 1 (1989), 32-42.

[11] Kerstin Dautenhahn. 1998. The art of designing socially intelligent agents Science, fiction, and the human in the loop. Applied artificial intelligence 12, 7-8 (1998), 573-617.

[12] Vania Dimitrova. 2003. STyLE-OLM: Interactive open learner modelling. International Journal of Artificial Intelligence in Education 13, 1 (2003), 35-78.

[13] Magy Seif El-Nasr, Anders Drachen, and Alessandro Canossa. 2016. Game analytics. Springer.

[14] Jerry Alan Fails and Dan R Olsen Jr. 2003. Interactive machine learning. In Proceedings of the 8th international conference on Intelligent user interfaces. 39-45.

[15] Guo Freeman and Donghee Yvette Wohn. 2017. eSports as an emerging research context at CHI: Diverse perspectives on definitions. In Proceedings of the $2017 \mathrm{CH}$ conference extended abstracts on human factors in computing systems. 1601-1608.

[16] Johannes Fürnkranz. 2007. Recent advances in machine learning and game playing. ÖGAI fournal 26, 2 (2007), 19-28.

[17] Wayne D Gray. 2017. Game-XP: Action games as experimental paradigms for cognitive science.

[18] George A Hazelrigg. 1998. A framework for decision-based engineering design. (1998).

[19] Victoria Hodge, Sam Devlin, Nick Sephton, Florian Block, Anders Drachen, and Peter Cowling. 2017. Win prediction in esports: Mixed-rank match prediction in multi-player online battle arena games. arXiv preprint arXiv:1711.06498 (2017).

[20] Victoria J Hodge, Sam Michael Devlin, Nicholas John Sephton, Florian Oliver Block, Peter Ivan Cowling, and Anders Drachen. 2019. Win prediction in multiplayer esports: Live professional match prediction. IEEE Transactions on Games (2019).

[21] Andreas Holzinger. 2016. Interactive machine learning for health informatics: when do we need the human-in-the-loop? Brain Informatics 3, 2 (2016), 119-131.

[22] Max Jaderberg, Wojciech M Czarnecki, Iain Dunning, Luke Marris, Guy Lever Antonio Garcia Castaneda, Charles Beattie, Neil C Rabinowitz, Ari S Morcos, Avraham Ruderman, et al. 2019. Human-level performance in 3D multiplayer games with population-based reinforcement learning. Science 364, 6443 (2019), 859-865.

[23] Andreas Larsson. 2003. Making sense of collaboration: the challenge of thinking together in global design teams. In Proceedings of the 2003 international ACM SIGGROUP conference on Supporting group work. 153-160.
[24] Marlos C Machado, Eduardo PC Fantini, and Luiz Chaimowicz. 2011. Player modeling: Towards a common taxonomy. In 2011 16th international conference on computer games (CGAMES). IEEE, 50-57.

[25] Marlos C Machado, Eduardo PC Fantini, and Luiz Chaimowicz. 2011. Player Modeling: What is it? How to do it? Proceedings of SBGames (2011).

[26] Keith Millis, Carol Forsyth, Patricia Wallace, Arthur C Graesser, and Gary Timmins. 2017. The impact of game-like features on learning from an intelligent tutoring system. Technology, Knowledge and Learning 22, 1 (2017), 1-22.

[27] Truong-Huy D Nguyen, Shree Subramanian, Magy Seif El-Nasr, and Alessandro Canossa. 2014. Strategy Detection in Wuzzit: A Decision Theoretic Approach. In International Conference on Learning ScienceWorkshop on Learning Analytics for Learning and Becoming a Practice.

[28] David Sousa Nunes, Pei Zhang, and Jorge Sá Silva. 2015. A survey on human-inthe-loop applications towards an internet of all. IEEE Communications Surveys \& Tutorials 17, 2 (2015), 944-965.

[29] Omnicoach.gg. 2021. Omnicoach.GG - AI Coach with Video Guides for Gaming. https://www.omnicoach.gg/. [Online; accessed 12-Feb-2021]

[30] Ismael Pedraza-Ramirez, Lisa Musculus, Markus Raab, and Sylvain Laborde. 2020. Setting the scientific stage for esports psychology: a systematic review. International Review of Sport and Exercise Psychology 13, 1 (2020), 319-352.

[31] Matthew A Pluss, Kyle JM Bennett, Andrew R Novak, Derek Panchuk, Aaron J Coutts, and Job Fransen. 2019. Esports: the chess of the 21st century. Frontiers in psychology 10 (2019), 156.

[32] David Premack and Guy Woodruff. 1978. Does the chimpanzee have a theory of mind? Behavioral and brain sciences 1, 4 (1978), 515-526.

[33] Sebastian Risi and Mike Preuss. 2020. From chess and atari to starcraft and beyond: How game AI is driving the world of AI. KI-Künstliche Intelligenz 34, 1 (2020), 7-17.

[34] Senpai.gg. 2021. SenpAI.GG - AI Coach with Video Guides for Gaming. https: //www.senpai.gg/. [Online; accessed 12-Feb-2021].

[35] Murtuza Shergadwala, Ilias Bilionis, Karthik N Kannan, and Jitesh H Panchal. 2018. Quantifying the impact of domain knowledge and problem framing on sequential decisions in engineering design. Journal of Mechanical Design 140, 10 (2018).

[36] Murtuza Shergadwala, Ilias Bilionis, and Jitesh H Panchal. 2018. Students as sequential decision-makers: Quantifying the impact of problem knowledge and process deviation on the achievement of their design problem objective. In International Design Engineering Technical Conferences and Computers and Information in Engineering Conference, Vol. 51784. American Society of Mechanical Engineers, V003T04A011.

[37] Murtuza Shergadwala, Karthik N Kannan, and Jitesh H Panchal. [n.d.]. Understanding the impact of expertise on design outcome: An approach based on concept inventories and item response theory. In ASME 2016 International Design Engineering Technical Conferences and Computers and Information in Engineering Conference. American Society of Mechanical Engineers Digital Collection.

[38] Herbert A Simon. 1988. The science of design: Creating the artificial. Design Issues (1988), 67-82.

[39] Gita Sukthankar, Christopher Geib, Hung Hai Bui, David Pynadath, and Robert P Goldman. 2014. Plan, activity, and intent recognition: Theory and practice. Newnes.

[40] H Jaap van den Herik, HHLM Donkers, and Pieter HM Spronck. 2005. Opponent modelling and commercial games. Proc. IEEE (2005), 15-25.

[41] Dakuo Wang, Elizabeth Churchill, Pattie Maes, Xiangmin Fan, Ben Shneiderman, Yuanchun Shi, and Qianying Wang. 2020. From Human-Human Collaboration to Human-AI Collaboration: Designing AI Systems That Can Work Together with People. In Extended Abstracts of the 2020 CHI Conference on Human Factors in Computing Systems. 1-6.

[42] Tian Wang. 2018. Predictive Analysis on eSports Games: A Case Study on League of Legends (LoL) eSports Tournaments. (2018).

[43] Thomas Weiss and Sabrina Schiele. 2013. Virtual worlds in competitive contexts: Analyzing eSports consumer needs. Electronic Markets 23, 4 (2013), 307-316.

[44] Georgios N Yannakakis, Pieter Spronck, Daniele Loiacono, and Elisabeth André. 2013. Player modeling. (2013).

[45] Georgios N Yannakakis and Julian Togelius. 2015. Experience-driven procedural content generation. In 2015 International Conference on Affective Computing and Intelligent Interaction (ACII). IEEE, 519-525.

[46] Wako Yoshida, Ray J Dolan, and Karl J Friston. 2008. Game theory of mind. PLoS Comput Biol 4, 12 (2008), e1000254 